\title{
Asymmetry of Convex Bodies of Constant Width
}

\author{
HaiLin Jin • Qi Guo
}

Received: 19 November 2010 / Revised: 4 May 2011 / Accepted: 10 June 2011 /

Published online: 13 July 2011

(C) Springer Science+Business Media, LLC 2011

\begin{abstract}
The symmetry of convex bodies of constant width is discussed in this paper. We proved that for any convex body $K \subset \mathbb{R}^{n}$ of constant width, $1 \leq \operatorname{as}_{\infty}(K) \leq$ $\frac{n+\sqrt{2 n(n+1)}}{n+2}$, where $\operatorname{as}_{\infty}(\cdot)$ denotes the Minkowski measure of asymmetry for convex bodies. Moreover, the equality holds on the left-hand side precisely iff $K$ is an Euclidean ball and the upper bounds are attainable, in particular, if $n=3$, the equality holds on the right-hand side if $K$ is a Meissner body.
\end{abstract}

Keywords Convex body of constant width · Asymmetry measure · Meissner's body

\section{Introduction}

Measures of (central) symmetry or, as we prefer, asymmetry for convex bodies have been extensively investigated (see [11, 13]). Among these researches, for a given asymmetry measure, it is a popular topic to determine the extremal bodies, i.e., the bodies with maximal asymmetry measure, in some class of convex bodies. For instance, for many known asymmetry measures, circles are most symmetric and Reuleaux triangles are most asymmetric among convex bodies of constant width in $\mathbb{R}^{2}$ (see [4, 5, 7, 8, 10]). Recently, in [12] we showed that this is also valid for the well known Minkowski measure (for the definition see below) (see [12]). Concretely, for any 2-dimensional convex body $K$ of constant width,

$$
1 \leq \operatorname{as}_{\infty}(K) \leq \frac{\sqrt{3}+1}{2}
$$

H.L. Jin · Q. Guo ( $\varangle)$

The Department of Mathematics, Suzhou University of Technology and Science, Suzhou, 215009

China

e-mail: guoqi@mail.usts.edu.cn

H.L. Jin

e-mail: ahjhl@sohu.com 
where $\operatorname{as}_{\infty}(\cdot)$ denotes the Minkowski measure of asymmetry for convex bodies. Moreover, equality holds on the left-hand side precisely iff $K$ is a circular disc, and on the right-hand side precisely iff $K$ is a Reuleaux triangle.

In this paper, we discuss the symmetry of convex bodies of constant width in $\mathbb{R}^{n}$. The main result is the following

Main Theorem If $K \subset \mathbb{R}^{n}$ is a convex body of constant width, then

$$
1 \leq \operatorname{as}_{\infty}(K) \leq \frac{n+\sqrt{2 n(n+1)}}{n+2} .
$$

Moreover, the equality holds on the left-hand side precisely iff $K$ is an Euclidean ball and the upper bounds are attainable, in particular, if $n=3$, the equality holds on the right-hand side if $K$ is a Meissner body.

\section{Preliminary}

$\mathbb{R}^{n}$ denotes the usual $n$-dimensional Euclidean space with the canonical inner product $\langle\cdot, \cdot\rangle$. A bounded closed convex set $C \subset \mathbb{R}^{n}$ is called a convex body (a convex domain for $n=2$ ) if it has non-empty interior (int for brevity). The family of all convex bodies in $\mathbb{R}^{n}$ is denoted by $\mathcal{K}^{n}$. Other notation and terms are as in [15].

A convex body $K$ is said to be of constant width $\omega$ if its projection on any straight line is a segment of universal length $\omega>0$, which is equivalent to the geometrical fact that any two parallel support hyperplanes of $K$ are always separated by a distance $\omega$. Notice that the width $\omega$ of a constant with body is clearly its diameter. The convex bodies of constant width in $\mathbb{R}^{2}$ and $\mathbb{R}^{3}$ are also called orbiforms and spherof orms, respectively. Euclidean balls are obvious bodies of constant width, however, there are many others (see $[1,6,14])$. We denote by $\mathcal{W}^{n}$ the set of all $n$-dimensional convex bodies of constant width.

Convex bodies of constant width have many interesting properties and applications which have gained much attention in the history, e.g., orbiforms were popularly studied during the nineteenth century and later, particularly by F. Reuleaux, whose name is now attached to the orbiforms obtained by intersecting a finite number of disks of equal radii. In $\mathbb{R}^{3}$, Meissner tetrahedrons may be the most famous spheroforms. Mathematicians guess Meissner tetrahedrons have the minimal volume among all spheroforms with the same width.

Given a convex body $C \in \mathcal{K}^{n}$ and $x \in \operatorname{int}(C)$, for a hyperplane $H$ through $x$ and the pair of support hyperplanes $H_{1}, H_{2}$ (of $C$ ) parallel to $H$, let $\gamma(H, x)$ be the ratio, not less than 1 , in which $H$ divides the distance between $H_{1}$ and $H_{2}$. Denote

$$
\gamma(C, x)=\max \{\gamma(H, x) \mid H \ni x\}
$$

and define the Minkowski measure $\operatorname{as}_{\infty}(C)$ of asymmetry of $C$ by (see [11])

$$
\operatorname{as}_{\infty}(C)=\min _{x \in \operatorname{int}(C)} \gamma(C, x) .
$$


A point $x \in \operatorname{int}(C)$ satisfying $\gamma(C, x)=\operatorname{as}_{\infty}(C)$ is called a critical point (of $C$ ). The set of all critical points of $C$ is denoted by $C^{*}$. It is known that $C^{*}$ is a non-empty convex set ([13]).

There is an equivalent definition of Minkowski measure (see [11,13]): Let $C \in \mathcal{K}^{n}$ and $x \in \operatorname{int}(C)$. For a chord $l$ of $C$ through $x$, let $\gamma_{1}(l, x)$ be the ratio, not less than 1 , in which $x$ divides the length of $l$, and denote $\gamma_{1}(C, x)=\max \left\{\gamma_{1}(l, x) \mid l \ni x\right\}$, then the Minkowski measure $\operatorname{as}_{\infty}(C)=\min _{x \in \operatorname{int}(C)} \gamma_{1}(C, x)$. A chord $l$ satisfying $\gamma_{1}(l, x)=\operatorname{as}_{\infty}(C)$ is called a critical chord (of $C$ ).

Denote for any $x \in \operatorname{int}(C)$,

$$
S_{C}(x)=\left\{p \in \operatorname{bd}(C) \mid \text { the chord } p q \ni x \text { and } \frac{x p}{x q}=\gamma_{1}(C, x)\right\},
$$

where bd denotes the boundary, and $p q$ denotes the segment with endpoints $p, q$ or its length alternatively if no confusing is caused. It is proved that $S_{C}(x) \neq \phi([13])$.

The following is a list of some properties of the Minkowski measure of asymmetry (see [13] for proofs).

Property 1 If $C \in \mathcal{K}^{n}$, then $1 \leq \operatorname{as}_{\infty}(C) \leq n$. Equality holds on the left-hand side precisely iff $C$ is central, and on the right-hand side precisely iff $C$ is a simplex.

Property 2 For $C \in \mathcal{K}^{n}, \operatorname{as}_{\infty}(C)+\operatorname{dim} C^{*} \leq n$, where $\operatorname{dim}$ means dimension.

Property 3 Given $x \in \operatorname{ri}\left(C^{*}\right)$, the relative interior of $C^{*}$, then for any $y \in S_{C}(x)$,

$$
y+\frac{\operatorname{as}_{\infty}(C)+1}{\operatorname{as}_{\infty}(C)}\left(C^{*}-y\right) \subset \operatorname{bd}(C) \text { and } y \in S_{C}\left(x^{\prime}\right) \quad \text { for } \forall x^{\prime} \in C^{*} .
$$

This property shows that the set $S_{C}(x)$ does not vary as $x$ ranges over $\operatorname{ri}\left(C^{*}\right)$. We denote this set by $C^{\dagger}$.

Property $4 C^{\dagger}$ contains at least as $_{\infty}(C)+1$ points.

\section{The Critical Sets of Convex Bodies of Constant Width}

We have known that the critical points of a convex bodies may not be unique, but for a convex body of constant width we have the following

Proposition 1 For any $K \in \mathcal{W}^{n}, K^{*}$ is a singleton.

In order to prove the proposition, we need the following lemmas 
Lemma 1 ([9]) If $C^{*}$ is not a singleton for some $C \in \mathcal{K}^{n}$, then $\operatorname{bd}(C)$ contains three different segments parallel to a segment contained in $C^{*}$.

Proof Suppose $C^{*}$ is not a singleton, then by the convexity of $C^{*}$, there exists segment $L_{1} \subset C^{*}$. Thus, if for any $y \in C^{\dagger}$, denote a segment $L_{1}(y):=y+a\left(L_{1}-y\right)$, where $a=\frac{\operatorname{as}_{\infty}(C)+1}{\operatorname{as}_{\infty}(C)}$, then by Property $3, L_{1}(y) \subset \operatorname{bd}(C) . L_{1}(y)$ is obviously parallel to $L_{1}$. Since by Property 4 , there are at least three points in $C^{\dagger}$ (notice that if $\operatorname{as}_{\infty}(C)=1$, then $C^{\dagger}$ contains infinitely many points, if $\operatorname{as}_{\infty}(C)>1$, then $\operatorname{as}_{\infty}(C)+1>2$ ), the proof is finished.

Lemma 2 ([14]) If $K \in \mathcal{W}^{n}$, then $K$ is strictly convex, i.e., bd(K) contains no segments.

The proof of this lemma in [14] appears a little cumbersome, so we include a proof here.

Proof If $K$ is not strictly convex, then there exists a segment $p q \subset \operatorname{bd}(K)$. Let $H$ be a hyperplane such that $H$ supports $K$ and $p q \subset H$ and let $H^{\prime}$ be the support hyperplane of $K$ which is opposite and parallel to $H$, then for each $y \in \operatorname{bd}(K) \cap H^{\prime}$, at least one of $p y$ and $q y$ is bigger than the width (i.e., the distance between $H$ and $H^{\prime}$ ) of $K$, a contradiction!

Proof of Proposition 1 It is a simple consequence of Lemma 1 and Lemma 2.

In general the insphere and the circumsphere of a convex body are not concentric. If, however, $K$ is a convex body of constant width $\omega$, then the insphere and circumsphere are concentric and their radii, denoted by $r(K)$ and $R(K)$, respectively, satisfy $r(K)+R(K)=\omega($ see $[2,6])$.

Here, we shall show further that the common center is also the unique critical point of a convex body of constant width.

Theorem 1 Let $K \in \mathcal{W}^{n}$ with constant width $\omega$. Then $x_{0}$ is the critical point of $K$ iff $x_{0}$ is the center of circumscribed sphere of $K$ and iff $x_{0}$ is the center of inscribed sphere of $K$. Moreover, $r(K)=\frac{1}{1+\mathrm{as}_{\infty}(K)} \omega$ and $R(K)=\frac{\mathrm{as}_{\infty}(K)}{1+\mathrm{as}_{\infty}(K)} \omega$.

Even if it was already proved that the insphere and the circumsphere of a convex body of constant width are concentric [2, 6, 14], but for completeness, we include also a simple proof here. In order to prove Theorem 1 , we need some more lemmas.

Lemma 3 ([13]) If $C \in \mathcal{K}^{n}, x \in \operatorname{int}(C)$ and $p q$ is a chord (of $C$ ) through $x$ with $p \in S_{C}(x)$, (i.e., $\frac{p x}{x q}=\gamma_{1}(C, x)$ ), then there exist two parallel hyperplanes $H_{1}, H_{2}$ supporting $C$ at $p, q$, respectively.

Corollary 1 If $K \in \mathcal{W}^{n}$ with constant width $\omega, x \in \operatorname{int}(K)$ and $p q$ is a chord (of $K$ ) through $x$ with $p \in S_{C}(x)$, then $p q=\omega$. 
Proof By Lemma 3, there exist two parallel hyperplanes $H_{1}, H_{2}$ supporting $K$ at $p, q$, respectively, so $\omega \leq p q$. But since $\omega$ is the diameter of $K, \omega \geq p q$. Therefore $p q=\omega$.

For $C \in \mathcal{K}^{n}, x \in C$, denote $r_{x}(C):=\max \{\lambda \geq 0 \mid x+\lambda B \subset C\}$ and $R_{x}(C):=$ $\min \{\lambda \geq 0 \mid C \subset x+\lambda B\}$, where $B \subset R^{n}$ is the Euclidean unit ball. Then clearly $r(C)=\max _{x \in \operatorname{int}(C)} r_{x}(C)$ and $R(C)=\min _{x \in \operatorname{int}(C)} R_{x}(C)$.

Lemma 4 If $K \in \mathcal{W}^{n}$ with constant width $\omega$ and $x \in \operatorname{int}(K)$, denote by $B_{x}$ the ball with center at $x$ and radius $\frac{\gamma_{1}(K, x)}{1+\gamma_{1}(K, x)} \omega$, then $K \subset B_{x}$, more precisely, $R_{x}(K)=$ $\frac{\gamma_{1}(K, x)}{1+\gamma_{1}(K, x)} \omega$.

Proof Let $p q$ be a chord (of $K$ ) through $x$ with $p \in S_{C}(x)$. By Lemma 3, there exist two parallel hyperplanes $H_{1}, H_{2}$ supporting $K$ at $p, q$, respectively. By Corollary 1 , $p x+x q=p q=\omega$ and in turn $p x=\frac{\gamma_{1}(K, x)}{1+\gamma_{1}(K, x)} \omega$. Letting $p^{\prime} q^{\prime}$ be any other chord through $x$, then by $\frac{p^{\prime} x}{p^{\prime} q^{\prime}-p^{\prime} x}=\frac{p^{\prime} x}{q^{\prime} x} \leq \gamma_{1}(K, x)$, we get

$$
p^{\prime} x \leq \frac{\gamma_{1}(K, x)}{1+\gamma_{1}(K, x)} p^{\prime} q^{\prime} \leq \frac{\gamma_{1}(K, x)}{1+\gamma_{1}(K, x)} \omega
$$

which implies $p^{\prime} \in B_{x}$ and further $K \subset B_{x}$ by the arbitrariness of $p^{\prime} \in \operatorname{bd}(K)$.

Finally, $p \in \operatorname{bd}(K)$ and $p x=\frac{\gamma_{1}(K, x)}{1+\gamma_{1}(K, x)} \omega$ imply $R_{x}(K)=\frac{\gamma_{1}(K, x)}{1+\gamma_{1}(K, x)} \omega$.

Lemma 5 If $K \in \mathcal{W}^{n}$ with constant width $\omega$ and $x \in \operatorname{int}(K)$, denote by $B_{x}^{*}$ the ball with center at $x$ and radius $\frac{\omega}{1+\gamma_{1}(K, x)}$, then $B_{x}^{*} \subset K$, more precisely, $r_{x}(K)=$ $\frac{\omega}{1+\gamma_{1}(K, x)}$.

Proof Let $q \in \operatorname{bd}(K)$ satisfy $r_{x}(K)=q x$ and let $H_{q}$ be a hyperplane supporting $K$ at $q$. Then $x q$ is orthogonal to $H_{q}$ since $H_{q}$ supports the ball with center at $x$ and radius $r_{x}(K)$ at $q$ as well.

Let $H_{p}$ be the hyperplane, parallel to $H_{q}$, supporting $K$ at $p$, then $p q$ passes through $x$ since $p q$ must be orthogonal to $H_{q}$ as well (for otherwise, we would have $p q>\omega$, a contradiction to the fact that $\omega$ is the diameter of $K)$.

Next, we prove that $\gamma_{1}(K, x)=\frac{p x}{q x}$. Let $p^{\prime} q^{\prime} \ni x$ be a chord of $K$. Since $q^{\prime} x \geq q x$ by the definition of $r_{x}(K)$, we get $p^{\prime} x \leq p x$ (observing that $p^{\prime} x+q^{\prime} x \leq p x+q x=$ $\omega)$, which leads to $\frac{p x}{q x} \geq \frac{p^{\prime} x}{q^{\prime} x}$ and further $\gamma_{1}(K, x)=\frac{p x}{q x}$. Thus, $\gamma_{1}(K, x)=\frac{\omega-r_{x}(K)}{r_{x}(K)}$ and in turn $r_{x}(K)=\frac{\omega}{1+\gamma_{1}(K, x)}$.

Proof of Theorem 1 Let $x_{0}$ be the (unique) critical point of $K$, then $\gamma_{1}\left(K, x_{0}\right)<$ $\gamma_{1}(K, x)$ for any $x \in \operatorname{int}(K) \backslash\left\{x_{0}\right\}$. Thus by Lemma 4 and Lemma $5, R_{x_{0}}(K)<$ $R_{x}(K)$ and $r_{x_{0}}(K)>r_{x}(K)$ for any $x \in \operatorname{int}(K) \backslash\left\{x_{0}\right\}$, which means that $R(K)=$ $R_{x_{0}}(K), r(K)=r_{x_{0}}(K)$ and $x_{0}$ is the common center of circumscribed and inscribed sphere of $K$. 
Let $x_{0}$ be the center of circumscribed sphere, then

$$
\begin{aligned}
\frac{\gamma_{1}\left(K, x_{0}\right)}{1+\gamma_{1}\left(K, x_{0}\right)} \omega & =R_{x_{0}}(K)=\min _{x \in \operatorname{int}(K)} R_{x}(K) \\
& =\min _{x \in \operatorname{int}(K)} \frac{\gamma_{1}(K, x)}{1+\gamma_{1}(K, x)} \omega=\frac{\min _{x \in \operatorname{int}(K)} \gamma_{1}(K, x)}{1+\min _{x \in \operatorname{int}(K)} \gamma_{1}(K, x)} \omega,
\end{aligned}
$$

which leads to $\gamma_{1}\left(K, x_{0}\right)=\min _{x \in \operatorname{int}(K)} \gamma_{1}(K, x)$, i.e., $x_{0}$ is the critical point of $K$.

The same argument works as well for the case when $x_{0}$ is the center of inscribed sphere.

Finally, let $x_{0}$ be the critical point of $K$, then what just proved above, together with Lemma 4 and Lemma 5, gives $R(K)=R_{x_{0}}(K)=\frac{\mathrm{as}_{\infty}(K)}{1+\mathrm{as}_{\infty}(K)} \omega$ and $r(K)=r_{x_{0}}(K)=$ $\frac{1}{1+\operatorname{as}_{\infty}(K)} \omega$.

Remark 1 For $K \in \mathcal{W}^{n}$ with constant width $\omega$, from Lemma 4, Lemma 5, we see that for any $x \in \operatorname{int}(K), R_{x}(K)+r_{x}(K)=\omega$ and $\gamma_{1}(K, x)=\frac{R_{x}(K)}{r_{x}(K)}$, in particular, by Theorem $1, R(K)+r(K)=\omega$ and $\operatorname{as}_{\infty}(K)=\frac{R(K)}{r(K)}$.

\section{The Minkowski Measure of Meissner Tetrahedrons}

Meissner's tetrahedron is geometrically defined in the following way: consider a body $K$ obtained as the intersection of four balls of radius $\omega$ whose centers are the vertices of an (underlying) regular tetrahedron $e_{1} e_{2} e_{3} e_{4}$ (of edge length $e_{i} e_{j}=\omega, 1 \leq i \neq j \leq$ 4 ). Thus, the boundary of $K$ is composed of four pieces of balls connected by six arcs of circles. Surprisingly this set $K$ is not of constant width: geometrical considerations show that opposite circular edges are too far away. Hence, Meissner proposed to polish off three edges of $K$ in order to get a constant width body. Let $E$ be the union of three circular edges of $K$ which share a common vertex $e_{1}$. Then,

$$
M=\bigcap_{x \in E} B(x, \omega) \cap K
$$

is a convex body of constant width, called Meissner's Tetrahedron formed by $K$ ([14]). We mention also that there are some other ways of "polishing off" the above $K$ for constructing constant width bodies, and all the resulted bodies are called Meissner's bodies.

Proposition 2 Let $M$ be a Meissner tetrahedron formed by $K$ with constant width $\omega=e_{1} e_{2}$, then $\operatorname{as}_{\infty}(M)=\frac{3+2 \sqrt{6}}{5}$.

Proof We first mention an obvious fact that if a convex body is symmetric with respect to a hyperplane, then its critical set is symmetric with respect to the same hyperplane as well. Now, since $M$ is symmetric with respect to three planes containing $o, e_{1}, e_{i},(i=2,3,4)$, respectively, where $o$ is the critical point of the underlying regular tetrahedron, the unique critical point $x_{0}$ of $M$ must be on the line containing $o, e_{1}$ 
which is the intersection of these three planes. We assert that $x_{0}=o$. To see this, let $e_{1}^{*}$ be the intersection of the ray $\overrightarrow{e_{1} o}$ and $\operatorname{bd}(M)$. Then clearly,

$$
\gamma_{1}(M, o) \geq \frac{e_{1} o}{o e_{1}^{*}}=\frac{e_{1} o}{\omega-e_{1} o} .
$$

On the other hand, if denoting by $B\left(o, o e_{1}\right)$ the ball with center at $o$ and radius $o e_{1}$, then we see easily that $K \subset B\left(o, o e_{1}\right)$ which implies clearly $M \subset B\left(o, o e_{1}\right)$.

Now, for any chord $p q$ (of $M$ ) through $o$ and with $\frac{p o}{o q}=\gamma_{1}(M, o)$, by Corollary 1 , we have

$$
p q=\omega \quad \text { and } \quad \frac{p o}{o q}=\frac{p o}{\omega-p o} .
$$

Thus, since $o p \leq o e_{1}$ (for $\left.M \subset B\left(o, o e_{1}\right)\right)$, we get

$$
r_{1}(M, o)=\frac{p o}{\omega-p o} \leq \frac{e_{1} o}{\omega-e_{1} o}=\frac{e_{1} o}{o e_{1}^{*}},
$$

which implies clearly that $\gamma_{1}(M, o)=\frac{e_{1} o}{o e_{1}^{*}}=\frac{e_{1} o}{\omega-e_{1} o}$.

If $x_{0}$ locates on the segment $\left(e_{1}, o\right)$, let $e_{2}^{*}$ be the intersection of the ray $\overrightarrow{e_{2} x_{0}}$ and $\operatorname{bd}(M)$. Then $e_{2} e_{2}^{*}=\omega$ and $e_{2} o<e_{2} x_{0}$, and so

$$
\gamma_{1}\left(M, x_{o}\right) \geq \frac{e_{2} x_{0}}{x_{0} e_{2}^{*}}=\frac{e_{2} x_{0}}{\omega-e_{2} x_{0}}>\frac{e_{2} o}{\omega-e_{2} o}=\frac{e_{1} o}{\omega-e_{1} o}=\gamma_{1}(M, o),
$$

which contradicts to the fact that $x_{0}$ is the critical point of $M$.

Similarly, if $x_{0}$ locates on the segment $\left(o, e_{1}^{*}\right)$, then

$$
\gamma_{1}\left(M, x_{o}\right) \geq \frac{e_{1} x_{0}}{\omega-e_{1} x_{0}}>\frac{e_{1} o}{\omega-e_{1} o}=\gamma_{1}(M, o),
$$

a contradiction too. Therefore, $x_{0}=o$.

Now some elementary geometrical arguments and computations show that $o e_{1}=$ $\frac{\sqrt{6}}{4} \omega$ which in turn leads to $\operatorname{as}_{\infty}(M)=\gamma_{1}(M, o)=\frac{o e_{1}}{\omega-o e_{1}}=\frac{\sqrt{6} \omega / 4}{\omega-\sqrt{6} \omega / 4}=\frac{3+2 \sqrt{6}}{5}$.

Remark 2 We shall show in the next section that Proposition 2 holds for general Meissner's bodies as well.

\section{Proof of Main Theorem}

The following lemma from [3] will be needed

Lemma 6 If $C \subset \mathbb{R}^{n}$ is compact, then

$$
R(C) \leq \operatorname{diam}(C) \sqrt{\frac{n}{2(n+1)}},
$$

where diam $(C)$ denotes the diameter of $C$. 
Proof of Main Theorem For general $n$, the first inequality is trivial, so we prove the second only. Let $K$ be of constant width $\omega$. Then By Remark 1, Lemma 6,

$$
\operatorname{as}_{\infty}(K)=\frac{R(K)}{r(K)}=\frac{R(K)}{\omega-R(K)} \leq \frac{\sqrt{\frac{n}{2(n+1)}} \omega}{\omega-\sqrt{\frac{n}{2(n+1)}} \omega}=\frac{n+\sqrt{2 n(n+1)}}{n+2} .
$$

Since a convex body of constant width is centrally symmetric iff it is a ball and a convex body $C$ is centrally symmetric and iff $\operatorname{as}_{\infty}(C)=1$, we see that as $\operatorname{as}_{\infty}(K)=1$ iff $K$ is a ball.

In order to see that for general $n$ the upper bounds are attainable, recall from [6] that for $C \in \mathcal{K}^{n}$, there is at least one convex body $C^{\natural}$ of constant width satisfying that $C \subset C^{\natural}$ and $\operatorname{diam}(C)=\operatorname{diam}\left(C^{\natural}\right)$ (each of such bodies is called a completion of $C$ ). Letting $S$ be a regular simplex, we claim that $\operatorname{as}_{\infty}\left(S^{\natural}\right)=\frac{n+\sqrt{2 n(n+1)}}{n+2}$ for each completion $S^{\natural}$, in particular, for all Meissner's bodies when $n=3$.

In fact, writing $\operatorname{diam}(S)=\omega=\operatorname{diam}\left(S^{\natural}\right)$, we have $R\left(S^{\natural}\right) \leq \omega \sqrt{\frac{n}{2(n+1)}}$ which, together with the obvious fact that $R\left(S^{\natural}\right) \geq R(S)=\omega \sqrt{\frac{n}{2(n+1)}}$, shows $R\left(S^{\natural}\right)=$ $\omega \sqrt{\frac{n}{2(n+1)}}$, so it follows that $\operatorname{as}_{\infty}\left(S^{\natural}\right)=\frac{R\left(S^{\natural}\right)}{\omega-R\left(S^{\natural}\right)}=\frac{n+\sqrt{2 n(n+1)}}{n+2}$.

Remark 3 (i) By Main Theorem, $\operatorname{as}_{\infty}(K)<1+\sqrt{2}$ for all $n$ and all $K \in \mathcal{W}^{n}$.

(ii) For $n=2$, it was shown in [12] that the Reuleaux triangles are the only extremal bodies with the Minkowski measures $\frac{1+\sqrt{3}}{2}$. However, for $n \geq 3$, since the completions of $S$ are not unique, the extremal bodies for the upper bounds are not unique. Since so, we propose a question: whether or not are these completions of regular simplices the only extremal bodies?

Finally, as an application, we give an estimate of $\sup _{K \in \mathcal{W}^{n}} \delta(K, B)$, where $B$ is an $n$-dimensional Euclidean unit ball and

$$
\delta(C, D):=\inf \{|\alpha / \beta|: \beta C \subset \mathrm{T}(D)+x, \alpha C \supset \mathrm{T}(D)+y\}
$$

$\left(\mathrm{T}: \mathbb{R}^{n} \rightarrow \mathbb{R}^{n}\right.$ regular affine maps, $\left.x, y \in \mathbb{R}^{n}\right)$.

Theorem 2 For any $K \in \mathcal{W}^{n}, \delta(K, B)=\operatorname{as}_{\infty}(K)$.

Proof B. Grünbaum showed that as $\operatorname{s}_{\infty}(C)=\inf _{M \in \mathcal{M}^{n}} \delta(C, M)$ for any convex body $C$, where $\mathcal{M}^{n}$ denotes the family of all $n$-dimensional centrally symmetric convex bodies ( $c f$. Sects. 5 and 6 in [11]), which implies as ${ }_{\infty}(K) \leq \delta(K, B)$.

However, letting $x_{0}$ be the critical point of $K$, then by Theorem 1 , we have also

$$
x_{0}+r(K) B \subset K \subset x_{0}+R(K) B,
$$

which, together with Remark 1, implies that $\delta(K, B) \leq \frac{R(K)}{r(K)}=\operatorname{as}_{\infty}(K)$. Hence, $\delta(K, B)=\operatorname{as}_{\infty}(K)$.

Corollary 2 For any $K \in \mathcal{W}^{n}, 1 \leq \delta(K, B) \leq \frac{n+\sqrt{2 n(n+1)}}{n+2}$. 
Acknowledgements Project supported partly by NSF of Jiangsu Hight Education No. 08KJD110016, partly by USTS Innovation Funds for Graduates (2009).

\section{References}

1. Bayen, T., Lachand-Robert, T., Qudet, E.: Analytic parametrization of three-dimensional bodies of constant width. Arch. Ration. Mech. Anal. 186, 225-249 (2007)

2. Benson, R.V.: Euclidean Geometry and Convexity. McGraw-Hill, New York (1966)

3. Berger, M.: Geometry I. Springer, Berlin (1987)

4. Besicovitch, A.S.: Measures of asymmetry for convex curves, II. Curves of constant width. J. Lond. Math. Soc. 26, 81-93 (1951)

5. Chakerian, G.D.: Sets of constant width. Pac. J. Math. 19(1), 13-21 (1996)

6. Charkerian, G.D., Groemer, H.: Convex bodies of constant width. In: Convexity and Its Applications, pp. 49-96. Birkhäuser, Basel (1983)

7. Charkerian, G.D., Stein, S.K.: Bisected chords of a convex body. Arch. Math. 17, 561-565 (1966)

8. Eggleston, H.G.: Measures of asymmetry of convex curves of constant width and restricted radii of curvature. Q. J. Math. Oxford Ser. (2) 3, 63-72 (1952)

9. Ekstrom, E.: The critical set of a convex body. U.U.D.M., Project Report 2000:P7

10. Groemer, H., Wallen, L.J.: A measure of asymmetry for domains of constant width. Beitrage Algebra Geom. 42, 517-521 (2001)

11. Grünbaum, B.: Measure of convex sets. In: Convexity, Proceedings of Symposia in Pure Mathematics, vol. 7, pp. 233-270. American Math. Society, Providence (1963)

12. Jin, H.L., Guo, Q.: On the asymmetry for convex domains of constant width. Commun. Math. Res. 26(2), 176-182 (2010)

13. Klee, V.L. Jr.: The critical set of a convex set. Am. J. Math. 75, 178-188 (1953)

14. Lachand-Robert, T., Qudet, E.: Bodies of constant width in arbitrary dimension. http://www.lama. univ-savoie.fr/ lachand/pdfs/spheroforms.pdf

15. Schneider, R.: Convex Bodies: The Brunn-Minkowski Theory. Cambridge University Press, Cambridge (1993) 